\title{
Laparoscopically assisted ventriculoperitoneal shunt placement: a prospective randomized controlled trial
}

\author{
${ }^{*}$ Philippe Schucht, MD, ${ }^{1}$ Vanessa Banz, MD, PhD, ${ }^{2}$ Markus Trochsler, MD, ${ }^{2}$ Samuel Iff, MD, ${ }^{3}$ \\ Anna Katharina Krähenbühl, MD, ${ }^{1}$ Michael Reinert, MD, ${ }^{4}$ Jürgen Beck, MD, ${ }^{1}$ Andreas Raabe, MD, ${ }^{1}$ \\ Daniel Candinas, MD, ${ }^{2}$ Dominique Kuhlen, MD, ${ }^{5}$ and Luigi Mariani, MD ${ }^{6}$
}

\begin{abstract}
Departments of ${ }^{1}$ Neurosurgery and ${ }^{2}$ Visceral Surgery and Medicine, Inselspital, University of Bern; ${ }^{3}$ Department of Clinical Research, Clinical Trials Unit Bern, University of Bern; ${ }^{6}$ Department of Neurosurgery, University Hospital Basel; ${ }^{4}$ Department of Neurosurgery, Ospedale Cantonale di Lugano, Switzerland; and ${ }^{5}$ Department of Neurosurgery, Klinikum rechts der Isar, Technische Universität München, Germany
\end{abstract}

OBJECT In ventriculoperitoneal (VP) shunt surgery, laparoscopic assistance can be used for placement of the peritoneal catheter. Until now, the efficacy of laparoscopic shunt placement has been investigated only in retrospective and nonrandomized prospective studies, which have reported decreased distal shunt dysfunction rates in patients undergoing laparascopic placement compared with mini-laparotomy cohorts. In this randomized controlled trial the authors compared rates of shunt failure in patients who underwent laparoscopic surgery for peritoneal catheter placement with rates in patients who underwent traditional mini-laparotomy.

METHODS One hundred twenty patients scheduled for VP shunt surgery were randomized to laparoscopic surgery or mini-laparotomy for insertion of the peritoneal catheter. The primary endpoint was the rate of overall shunt complication or failure within the first 12 months after surgery. Secondary endpoints were distal shunt failure, overall complication/ failure, duration of surgery and hospitalization, and morbidity.

RESULTS The overall shunt complication/failure rate was 15\% (9 of 60 cases) in the laparoscopic group and $18.3 \%$ (11 of 60 cases) in the mini-laparotomy group ( $p=0.404)$. Patients in the laparoscopic group had no distal shunt failures; in contrast, $5(8 \%)$ of 60 patients in the mini-laparotomy group experienced distal shunt failure $(p=0.029)$. Intraoperative complications occurred in 2 patients (both in the laparoscopic group), and abdominal pain led to catheter removal in 1 patient per group. Infections occurred in 1 patient in the laparoscopic group and 3 in the mini-laparotomy group. The mean durations of surgery and hospitalization were similar in the 2 groups.

CONCLUSIONS While overall shunt failure rates were similar in the 2 groups, the use of laparoscopic shunt placement significantly reduced the rate of distal shunt failure compared with mini-laparotomy.

Clinical trial registration no.: NCT01739179 (clinicaltrials.gov)

http://thejns.org/doi/abs/10.3171/2014.9.JNS132791

KEY WORDS hydrocephalus; laparoscopy; shunt dislocation; shunt dysfunction; VP shunt

$\mathrm{V}$ ENTRICULOPERITONEAL (VP) shunting was first described at the beginning of the 20th century as a CSF diversionary procedure in patients with hydrocephalus. ${ }^{12}$ After the introduction of Silastic catheters in the $1960 \mathrm{~s},{ }^{2,23}$ this method became the treatment of choice for children and adults with communicating hydrocephalus. The average patient requiring VP shunt treatment will undergo at least 2 shunt revisions in the first 3 years, with some patients requiring more than 20 revisions within the 1 st year. ${ }^{22}$ Therefore, any technical improvement with a positive impact on the revision rate provides an obvious benefit for the patient, while simultaneously reducing the surgical burden and consequent medical costs.

Distal shunt failures-either due to improper placement or secondary dislocation of the distal catheter out of the

ABBREVIATIONS BMI = body mass index; NSAID = nonsteroidal anti-inflammatory drug; VP = ventriculoperitoneal.

SUBMITTED December 17, 2013. ACCEPTED September 25, 2014.

INCLUDE WHEN CITING Published online December 23, 2014; DOI: 10.3171/2014.9.JNS132791.

DISCLOSURE The authors have no competing interests. The research was not supported by any specific grant from any funding agency in the public, commercial, or not-for-profit sectors.

* Drs. Schucht and Banz contributed equally to this work. Drs. Kuhlen and Mariani share senior authorship. 
peritoneal cavity-have been reported in 10\%-30\% of cases of shunt failure. ${ }^{9,14,22}$ The way the catheter is inserted seems to play a major role in subsequent failure. The classic mini-laparotomy requires closing of the peritoneal entry point around the catheter, carrying the risk of catheter ligation or secondary dislocation into the subcutaneous tissue. Visualization of the peritoneal cavity through minilaparotomy is generally poor, and catheter placement in obese patients and patients with adhesions owing to previous abdominal surgery presents an additional challenge. . $^{1,33}$

An alternative to mini-laparotomy in patients undergoing VP shunt surgery is the laparoscopic placement of the peritoneal catheter, as described by Basauri and colleagues in $1993 .{ }^{5}$ Retrospective series have since suggested an advantage in terms of operation duration, length of hospital stay, and rate of distal shunt dysfunction (and thus potentially overall shunt dysfunction) compared with mini-laparotomy, with very low risk for surgical complications such as viscus perforations. $3,5,7,8,13,15,16,20,24,26,34$

Laparoscopic shunt placement is hypothesized to have several advantages over the classic mini-laparotomy: 1) the peritoneal wall is only punctured, rather than incised, making fixation of the catheter, and therefore the potential danger of catheter ligation, obsolete; 2) there is no subcutaneous pouch into which the catheter can slip; 3) insertion of the catheter and its placement in the lesser pelvis are visually controlled by the intraperitoneal camera, making misplacement virtually impossible; and 4) presence of CSF flow through the catheter can be visually controlled after final catheter placement.

Evidence for these possible advantages of laparoscopic surgery over mini-laparotomy for VP shunt placement has been, until now, based on nonrandomized studies, in which the inherent selection bias may have influenced the outcomes.

Hence, our aim was to test our hypothesis that the use of laparoscopic VP shunt surgery decreases the rate of shunt complications and failures compared with classic mini-laparotomy surgery in a prospective, randomized controlled trial.

\section{Methods}

This was a single-center, parallel-group, randomized controlled trial conducted at the Bern University Hospital, Bern, Switzerland, between March 2007 and May 2011. Eligible patients met these criteria: 1) were age 18 years or older, 2) had either newly diagnosed hydrocephalus necessitating VP shunt placement or shunt malfunction necessitating VP shunt revision and replacement of the peritoneal catheter, and 3) provided written informed consent. Exclusion criteria were pregnancy, inability to provide informed consent, estimated survival less than 12 months, inability to communicate, or a remote location that complicated follow-up (e.g., patients living abroad). Only patients who were eligible for inclusion on the basis of these criteria and were scheduled to receive a new shunt system were randomized. Data were collected perioperatively and at 6 weeks, 6 months, and 12 months after surgery. The outcomes of the trial and corresponding analyses were prespecified in the study protocol. The lo- cal ethics commission approved the study. We complied with CONSORT (Consolidated Standards of Reporting Trials, www.consort-statement.org) in reporting on this trial. This study was registered with the ClinicalTrials. gov database (http://clincaltrials.gov) and its registration number is NCT01739179.

\section{Outcomes}

The primary endpoint was the overall rate of shunt malfunction requiring revision during 12 months of follow-up. Secondary endpoints were: 1) rate of overall shunt failure at 6 weeks and at 6 months and rate of distal shunt malfunction requiring revision at 6 weeks, 6 months, and 12 months; 2) time to resumption of full oral food intake, defined as the time (in days) until the patients were able to eat a standard meal provided by the hospital kitchen; 3) analgesic (nonsteroidal anti-inflammatory drug [NSAID]) use as evaluated on Day 5 postoperative$1 y ; 4)$ duration of the operation, defined as the time from the first incision to closure of all incisions; 5) duration of hospital stay, defined as day of admission until discharge from the neurosurgical ward; 6) time to recovery of full mobility, defined as number of days until the patient could move around without any support; and 7) correct proximal and distal catheter positioning.

A brain CT scan was obtained following surgery to verify the correct positioning of the ventricular catheter in the frontal horn of the lateral ventricle. Anteroposterior and lateral cranio-abdominal radiographs were obtained using a Lodox low-dose X-ray scanner (Statscan Critical Imaging System, Lodox Systems) to check for integrity of the shunt and correct positioning of the distal tip of the catheter in the abdominal cavity, as well as to rule out kinking of the catheter. ${ }^{6}$ Patient screening and selection for study inclusion, data collection, and follow-up examinations were performed by neurosurgeons of the Bern University Hospital Department of Neurosurgery.

\section{Sample Size}

Based on previous literature, ${ }^{9,14,22}$ an overall shunt failure rate of $20 \%$ at 12 months was estimated for mini-laparotomy distal shunt placement. A reduction of the rate to $5 \%$ in the laparoscopic group was considered clinically relevant. Assuming a Type I error rate of 0.05, 60 patients were needed in each arm of the trial to detect a difference of this magnitude with $80 \%$ power in a 1-sided test.

In cases of suspected shunt failure, a head CT scan and a cranio-abdominal Lodox scan were performed to confirm the clinical suspicion and to identify catheter misplacement, dislocation, or disconnection. The reason for shunt failure was assessed based on these imaging studies and on intraoperative findings. CT and Lodox scans were repeated after revision surgery.

\section{Randomization}

Once a patient had been identified for inclusion and informed consent obtained, randomization was performed by the treating neurosurgeon on site using sealed, opaque, sequentially numbered envelopes containing the allocation information. The allocation schedule was based on computer-generated random numbers. Patients were as- 
signed on a 1:1 basis to mini-laparotomy or laparoscopic distal shunt placement.

\section{Surgical Technique \\ Proximal Shunt Placement}

Protocol required the proximal shunt to be inserted and positioned according to a previously established, standardized procedure. ${ }^{2}$ A single prophylactic dose of cefuroxime was administered intravenously at induction of anesthesia, at least 30-45 minutes prior to skin incision. Following skin incision, the ventricular catheter was inserted at Kocher's point, $12 \mathrm{~cm}$ posterior to the nasion and $3 \mathrm{~cm}$ from the midline, preferably on the right side. The catheter was tunneled to the designated valve site behind the ipsilateral ear. A thread, tunneled from the abdominal entry site toward the retroauricular area, was used to pull the catheter through to the abdomen. All patients received programmable valves. The brand of valves was changed-for reasons unrelated to the study-from Codman-Hakim programmable valves to Miethke programmable valves after the initial 53 cases.

The cranial part of surgery and the abdominal part in the mini-laparotomy group were performed by a boardcertified neurosurgeon or a senior resident under supervision. A board-certified visceral surgeon or senior resident under supervision performed the abdominal surgery in the laparoscopic arm of the study.

\section{Distal Shunt Placement in the Mini-Laparotomy Group}

A 5-cm right-sided paraumbilical skin incision was made, and the underlying tissues were separated to expose the deep fascia. A VP shunt catheter was tunneled from the cranial location to the abdominal entry point. Pumping the valve controlled for adequate CSF outflow. The catheter length was adjusted so that $25 \mathrm{~cm}$ of catheter remained in the peritoneal cavity. The deep fascia and peritoneum were incised, and the distal catheter tip was inserted into the peritoneal cavity in a craniocaudal direction. The catheter was fixed and the peritoneal opening closed by means of a purse-string suture.

\section{Distal Shunt Placement in the Laparoscopic Group}

A periumbilical incision was made to allow introduction of a $12-\mathrm{mm}$ camera trocar. In the later study stages a 5-mm camera became available, allowing for a smaller incision. The pneumoperitoneum was established either by use of the Veress needle or by means of a mini-laparotomy with the Hasson technique,$^{10}$ according to the preference of the surgeon. A 1-cm skin incision was made in the right upper abdominal quadrant. The distal catheter was subcutaneously tunneled from the cranial end and externalized at the skin incision. The peritoneum was punctured under direct laparoscopic vision by a Cystofix peel-away needle as is commonly used for suprapubic cystostomy (Fig. 1). ${ }^{4}$ The Cystofix needle was advanced into the abdomen and the distal shunt catheter introduced in a craniocaudal direction so that a minimum of $25 \mathrm{~cm}$ was placed intraperitoneally. Under direct camera-enabled vision, the valve was pumped to control adequate intraabdominal outflow of CSF. The Cystofix needle was subsequently removed, and the peritoneal entry point was checked for bleeding before removal of the laparoscope. ${ }^{9}$

\section{Statistical Analysis}

The overall rate of shunt malfunction was analyzed using Fisher's exact test. The analyses were based on an intention-to-treat approach, with data from all randomized patients analyzed in the group to which they were originally allocated. Kaplan-Meier survival curves were produced for visual description, and a univariate Cox regression model was fitted with treatment as the explanatory variable. The follow-up period in survival analysis was defined from the time of inclusion to the end of follow-up (12 months). A multivariate Cox model was fitted, correcting for the covariates age, body mass index (BMI), sex, primary etiology, primary shunt, American Society of Anesthesiologists (ASA) classification, operation time, and previous abdominal surgery. These variables were selected because of their clinical relevance. The covariates were used to adjust the outcomes in an adjusted logistic regression model. The proportional hazards assumption of all Cox models was assessed statistically. ${ }^{30}$

Secondary outcomes, including distal shunt dysfunction, were analyzed using Fisher's exact test, a logistic regression model for binary outcomes, or a linear regression model for continuous outcomes. As additional analyses, these outcomes were corrected for the additional explanatory variables. For participant characteristics, means and proportions of variables were calculated.

A $p$ value $<0.05$ was considered statistically significant. Data analyses were performed with Stata for Windows, version 12.0 (StataCorp LP).

Departures from the initial published protocol included: 1) adding the rate of distal shunt dysfunction as a secondary outcome (added after study start), 2) removing the secondary outcome variable of time to resumption of full oral food intake (which turned out to be impractical due to improper documentation of when a first "proper" meal was taken), 3) removing the secondary outcome variable of quality of life (because of missing data due to insufficient data collection), and 4) adding a secondary time-toevent analysis (all added during final analysis).

\section{Results}

A total of 174 eligible patients were screened for inclusion, 54 of whom were excluded according to inclusion/ exclusion criteria. The main reasons for exclusion were age $<18$ years $(\mathrm{n}=13)$, inability to communicate $(\mathrm{n}=$ $8)$, estimated survival $<12$ months $(n=8)$, failure to obtain written consent $(n=7)$, and foreseeable postoperative follow-up difficulties due to geographic considerations ( $\mathrm{n}$ $=6$ ). The remaining 120 patients were randomized in a 1:1 ratio. Figure 2 presents the disposition of participants throughout the trial. Sixty patients were allocated to laparoscopic surgery and 60 to mini-laparotomy. All patients in the mini-laparotomy group received treatment as allocated. Two patients in the laparoscopic group did not undergo laparoscopic surgery: one patient received a mini-laparotomy because the visceral surgeon on call was unavailable, and another patient refused intervention and follow-up after randomization. All patients were followed until the 12-month follow-up visit or death, except for the one patient who refused further participation in 

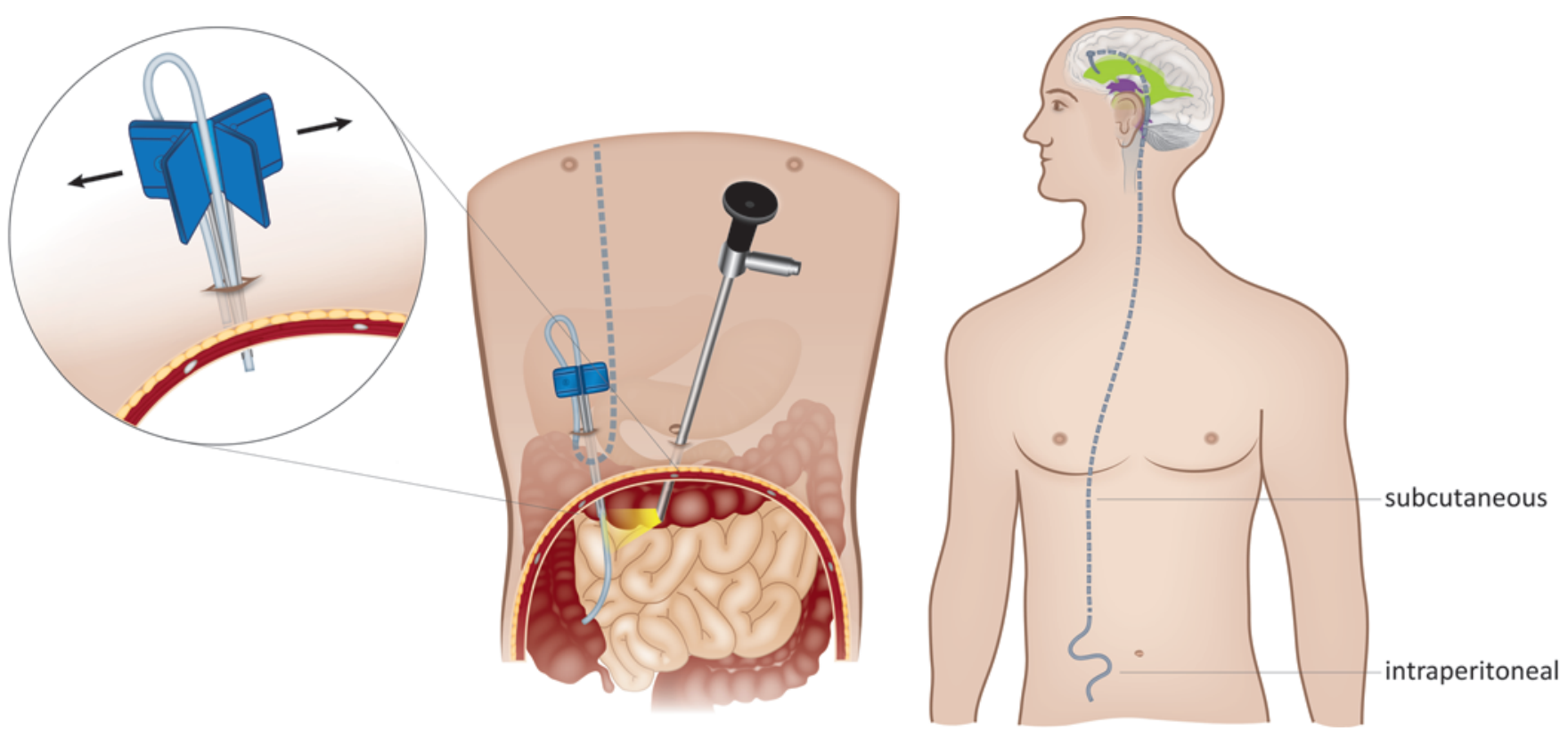

FIG. 1. Artist's illustration of laparoscopic VP shunt placement. A 5-mm optic trocar is inserted next to the navel after a sufficient pneumoperitoneum has been established. A peel-away needle (Cystofix, right upper abdominal quadrant, inset) is used to puncture the peritoneum. The distal shunt, which has been subcutaneously tunneled from the head toward the abdomen, is inserted through the Cystofix needle and placed into the peritoneal cavity under direct laparoscopic vision. Copyright Philippe Schucht. Published with permission.

the study. The majority of early deaths (within the first 6 weeks after VP shunt placement) occurred as a direct consequence of a subarachnoid hemorrhage or traumatic brain hemorrhage, whereas later deaths were mainly due to rapid cancer progression or recurrence.

The 2 groups had similar clinical characteristics at baseline, with the majority of patients receiving their first VP shunt (116 [97\%] of 120 patients). The indications for VP shunt placement were similar for the 2 groups, with subarachnoid hemorrhage and tumor being the most frequent causes of hydrocephalus (Table 1).

The overall rate of shunt failure was higher in the mini-laparotomy group at the 6-week follow-up assessment (Fig. 3), but this difference was not statistically significant at 12 months, with failure rates of $15.0 \%(\mathrm{n}=9)$ in the laparoscopic group and $18.3 \%(\mathrm{n}=11)$ in the minilaparotomy group ( $\mathrm{p}=0.404$, Fisher's exact test; Table 2$)$. Figures 3 and 4 show Kaplan-Meier curves of cumulative overall and distal shunt malfunction, respectively, requiring surgical intervention over the entire 12-month study period. The crude hazard ratio for overall shunt failure was 1.15 (95\% CI 0.44-2.97), but it was not statistically significant $(\mathrm{p}=0.78)$. The age-adjusted hazard ratio was 1.34 (95\% CI 0.51-3.53).

The rate of distal shunt failure differed significantly between the 2 groups, with 5 shunt revisions for distal shunt failure (8\%) in the mini-laparotomy group, compared with no distal shunt failures $(0 \%)$ in the laparoscopic group $(\mathrm{p}$ $=0.029$; Table 2). Patients with distal shunt failures were similar to patients without distal shunt failures in terms of age, duration of surgery, BMI, and previous abdominal surgery (Table 3 ). Three patients in the mini-laparotomy group (5\%) underwent revision for shunt infection compared with 1 patient (1.7\%) in the laparoscopic group. Cor- rect intracranial catheter positioning was confirmed in all patients in the mini-laparotomy group, whereas 2 patients in the laparoscopic group required an early surgical revision due to incorrect proximal catheter positioning $(\mathrm{p}=$ 0.244 , Fisher's exact test; Table 4). No disruption of the catheter in its subcutaneous run or abdominal dislocation and kinking were detected in the postoperative imaging in either group. One patient from each group underwent removal of the peritoneal catheter and conversion of the VP shunt to a ventriculoatrial shunt because of abdominal pain. The patient from the mini-laparotomy group had undergone 2 previous abdominal surgeries and then underwent shunt conversion surgery 24 days after the VP shunt surgery that was performed as part of the current study. This patient remained pain free after shunt conversion and was still pain free 12 months afterward. The patient from the laparoscopic group had undergone 6 previous abdominal surgeries, underwent additional surgery 132 days after initial VP shunt implantation, and reported persisting abdominal pain at 12 months' follow-up. We did not observe any abdominal pseudocyst formation.

Conversion of laparoscopic surgery to laparotomy was necessary in 2 patients due to accidental injuries to the small bowel (enterotomies). The injuries, which were due to adhesions of the bowel to the peritoneal wall, occurred during the initial approach to the peritoneum prior to insertion of the camera and the Cystofix peel-away needle. Both patients had undergone previous abdominal surgery: an operation for colorectal cancer in one case and previous explorative laparotomy for abdominal trauma in the other. In both patients the shunting procedure was aborted and a mini-laparotomy was performed to repair the lesion. One patient received a ventriculoatrial shunt 3 days later. The other patient experienced significant im- 


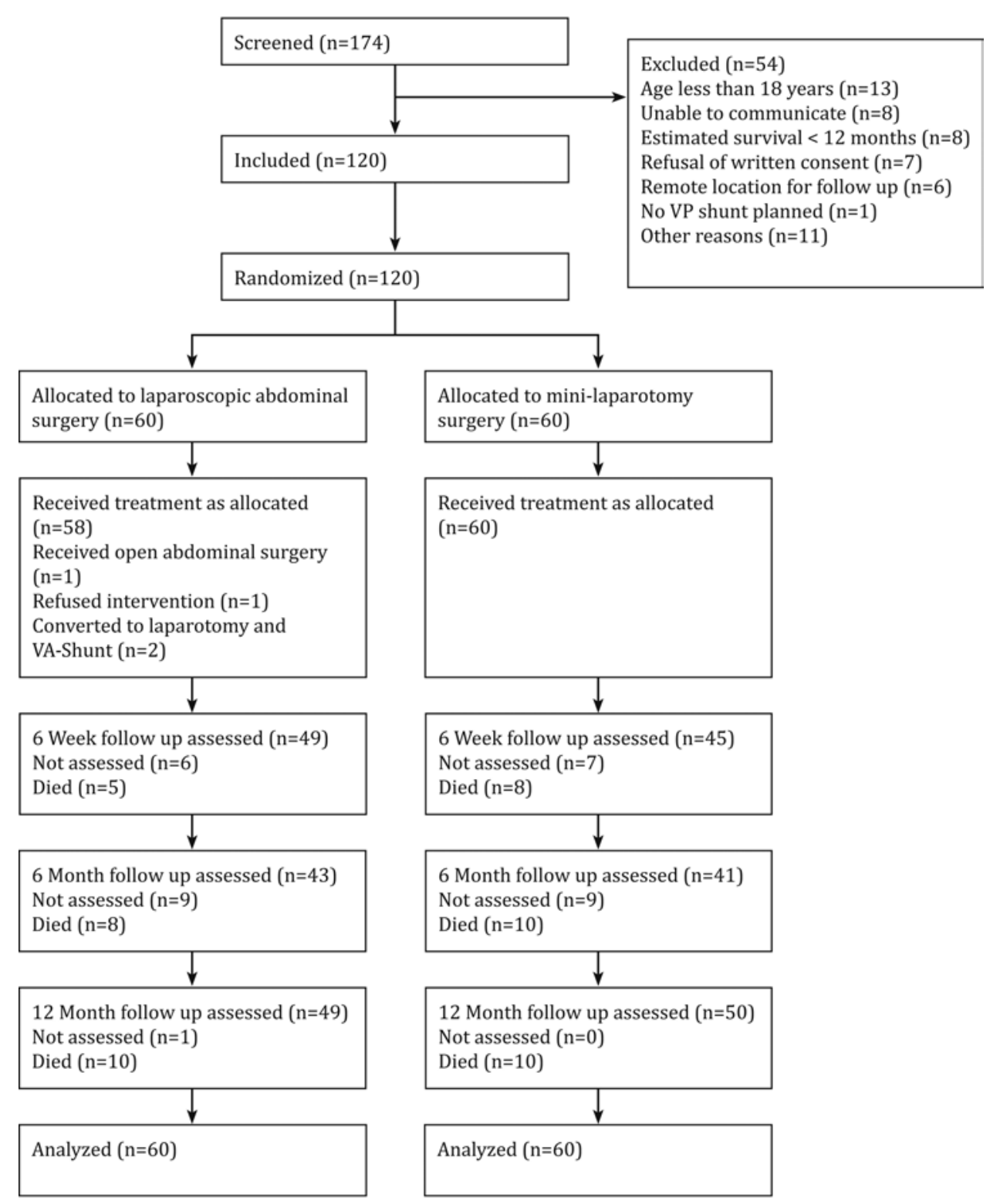

FIG. 2. Flow diagram. A total of 174 screened patients were initially eligible for study participation. After exclusion of 54 patients, a total of 120 patients were enrolled, 60 of whom were randomized to the laparoscopic surgery group and 60 to the mini-laparotomy group. $V A=$ ventriculoatrial.

provement in her hydrocephalic symptoms and did not undergo any further shunting procedures. These intraoperative complications were included in the final analysis of the overall rate of shunt failures.

Univariate analyses did not reveal statistically significant differences between the 2 groups in surgery duration, length of hospital stay, time to full mobility, time to resumption of complete solid food intake, or analgesic drug requirement (Table 4).

The unadjusted model showed an insignificant result for laparoscopy when shunt failure was used as the outcome (HR 1.14, $\mathrm{p}=0.8,95 \%$ CI 0.44-2.97). The predefined fully adjusted model failed due to the low number of events in relation to the number of parameters. Consequently, every parameter was tested with type of surgery only and was excluded if the $p$ value was above the sig- nificance level of 0.2. Only age at surgery remained in the final model (HR 0.96, $\mathrm{p}=0.03,95 \%$ CI 0.94-0.99), while the treatment effect on overall shunt failure remained insignificant (HR 1.33, $\mathrm{p}=0.6,95 \%$ CI 0.51-3.52).

\section{Discussion}

Although regarded as a routine neurosurgical procedure, implantation of a VP shunt is prone to complications and failures. The most frequent causes of shunt failure are malposition of the cranial or peritoneal catheter, shunt obstruction, and shunt infection. As yet, there is no consensus in the literature as to what defines a shunt complication or shunt failure. ${ }^{20} \mathrm{We}$ therefore analyze and discuss the various events, such as intraoperative complications, distal and proximal shunt failures, and other surgery-related morbidities, separately. 
TABLE 1. Summary of baseline characteristics of patients included in this study*

\begin{tabular}{lcc}
\hline & \multicolumn{2}{c}{ Group } \\
\cline { 2 - 3 } Baseline Characteristics & $\begin{array}{c}\text { Laparoscopic } \\
(\mathrm{n}=60)\end{array}$ & $\begin{array}{c}\text { Mini-Laparotomy } \\
(\mathrm{n}=60)\end{array}$ \\
\hline Mean age (yrs) & $56.4 \pm 17.5$ & $60.1 \pm 14.2$ \\
\hline Mean weight $(\mathrm{kg})$ & $70.7 \pm 14.4$ & $77.0 \pm 18.2$ \\
\hline Mean height $(\mathrm{cm})$ & $169.6 \pm 8.9$ & $170.2 \pm 8.7$ \\
\hline Mean BMI $\left(\mathrm{kg} / \mathrm{m}^{2}\right)$ & $24.7 \pm 4.4$ & $26.4 \pm 5.1$ \\
\hline Primary shunt & $57(95 \%)$ & $59(98 \%)$ \\
\hline Indication for shunt & & \\
\hline Subarachnoid bleeding & $23(38 \%)$ & $24(40 \%)$ \\
\hline Tumor & $12(20 \%)$ & $11(18 \%)$ \\
\hline Low-pressure hydrocephalus & $7(12 \%)$ & $9(15 \%)$ \\
\hline Bleeding & $8(13 \%)$ & $4(7 \%)$ \\
\hline Trauma & $4(7 \%)$ & $6(10 \%)$ \\
\hline Other & $6(10 \%)$ & $6(10 \%)$ \\
\hline Previous abdominal surgery & & \\
\hline No & $37(62 \%)$ & $43(72 \%)$ \\
\hline Yes & $22(37 \%)$ & $17(28 \%)$ \\
\hline Unknown & $1(2 \%)$ & $0(0 \%)$ \\
\hline ASA category & & \\
\hline I II & $18(30 \%)$ & $17(29 \%)$ \\
\hline III & $27(45 \%)$ & $30(50 \%)$ \\
\hline IV & $5(8 \%)$ & $8(13 \%)$ \\
\hline Missing & $10(17 \%)$ & $5(8 \%)$ \\
\hline
\end{tabular}

ASA = American Society of Anesthesiologists.

* Values represent numbers of patients (\%) unless otherwise indicated.

To our knowledge, this is the first prospective, randomized controlled trial comparing classic distal catheter placement through a mini-laparotomy with laparoscopic catheter insertion.

\section{Use of a Peel-Away Needle for Abdominal Catheter Insertion}

We used a Cystofix peel-away needle for insertion of the catheter into the abdominal cavity, visually controlled from a periumbilical camera. ${ }^{4}$ This elegant technique has several advantages compared with a mini-laparotomy approach. For insertion of the catheter, the peritoneum is not incised, as in the mini-laparotomy approach, but only punctured. Consequently, fixation of the catheter, which is known to be a risk factor for obstruction or secondary dislocation of the catheter in the mini-laparotomy approach, is not necessary. ${ }^{22}$ Additionally, there is no artificial subcutaneous pouch into which the catheter may slip, as may be encountered after a mini-laparotomy approach. Finally, CSF flow can be visually controlled after placement of the catheter into the peritoneum, ensuring prior to wound closure that no obstruction or disconnection occurred during shunt implantation.

\section{Overall Shunt Failure Rate}

There was an initial difference in the overall shunt failure rates in the 2 groups, but it had largely vanished by 12 months after shunt placement (Fig. 3), leaving a nonsignificant trend toward lower overall shunt failure in the laparoscopic compared with the mini-laparotomy group (15\% vs $18.3 \%$, respectively; $\mathrm{p}=0.404$ ). The initial difference in distal shunt failure rates was diluted by proximal shunt failures, infections, and other complications. The same effect was observed by Naftel et al. in the largest retrospective report to date; however, their statistically significant difference in verified distal shunt failure did not lead to a significant difference in overall shunt failure. ${ }^{20}$

\section{Distal Shunt Failure (Abdominal Malposition and Distal Obstruction)}

Distal shunt failures may occur due to malposition of the distal catheter during surgery or due to secondary dislocation out of the peritoneal cavity into the subcutaneous fat. Such distal shunt complications are a frequent cause of shunt revision, affecting up to $10 \%-30 \%$ of all patients. ${ }^{9,14,22}$ The lower rate of distal shunt failure after the laparoscopic procedure ( $0 \%$ distal failure, compared with $8 \%$ after mini-laparotomy) observed in the present study is in accordance with the literature. Bani et al. reported equally low distal failure rates in their consecutive series-0\% in their laparoscopic group and $8 \%$ in their mini-laparotomy group. ${ }^{4}$ Roth et al. reported distal shunt failure rates of $4 \%$ and $10.3 \%$ for laparoscopic and minilaparotomy methods, respectively, in a retrospective series. ${ }^{25}$ A prospective, nonrandomized study reported rates of $4 \%$ after laparoscopic assistance compared with $12 \%$ after mini-laparotomy. ${ }^{27}$ Hence, our study, in accordance with previous reports, shows that the problem of distal shunt failure is largely solved by the use of laparoscopy.

TABLE 2. Summary of all complications and VP shunt failures in the first 12 months after surgery

\begin{tabular}{lccc}
\hline \multicolumn{1}{c}{ Description } & \multicolumn{2}{c}{ No. of Patients $(\%)$} & \\
\cline { 2 - 3 } & Laparoscopic $(\mathrm{n}=60)$ & Mini-Laparotomy $(\mathrm{n}=60)$ & $\mathrm{p}$ Value \\
\hline Overall complications \& failures requiring surgical intervention & $9(15.0 \%)$ & $11(18.3 \%)$ & $0.40^{*}$ \\
\hline Distal shunt failure & $0(0 \%)$ & $5(8.3 \%)$ & $0.03^{*}$ \\
\hline Proximal shunt failure (failure of cranial catheter or valve) & $5(8.3 \%)$ & $2(3.3 \%)$ & $0.44 \dagger$ \\
\hline Infections & $1(1.7 \%)$ & $3(5.0 \%)$ & $0.62 \dagger$ \\
\hline Removal of peritoneal catheter due to abdominal pain & $1(1.7 \%)$ & $1(1.7 \%)$ & $1.00 \dagger$ \\
\hline Intraoperative complications & $2(3.3 \%)$ & $0(0.0 \%)$ & $0.50 \dagger$ \\
\hline
\end{tabular}

* One-sided Fisher's exact test, protocol-defined analysis.

$\dagger$ Two-sided Fisher's exact test, post-protocol analysis. 


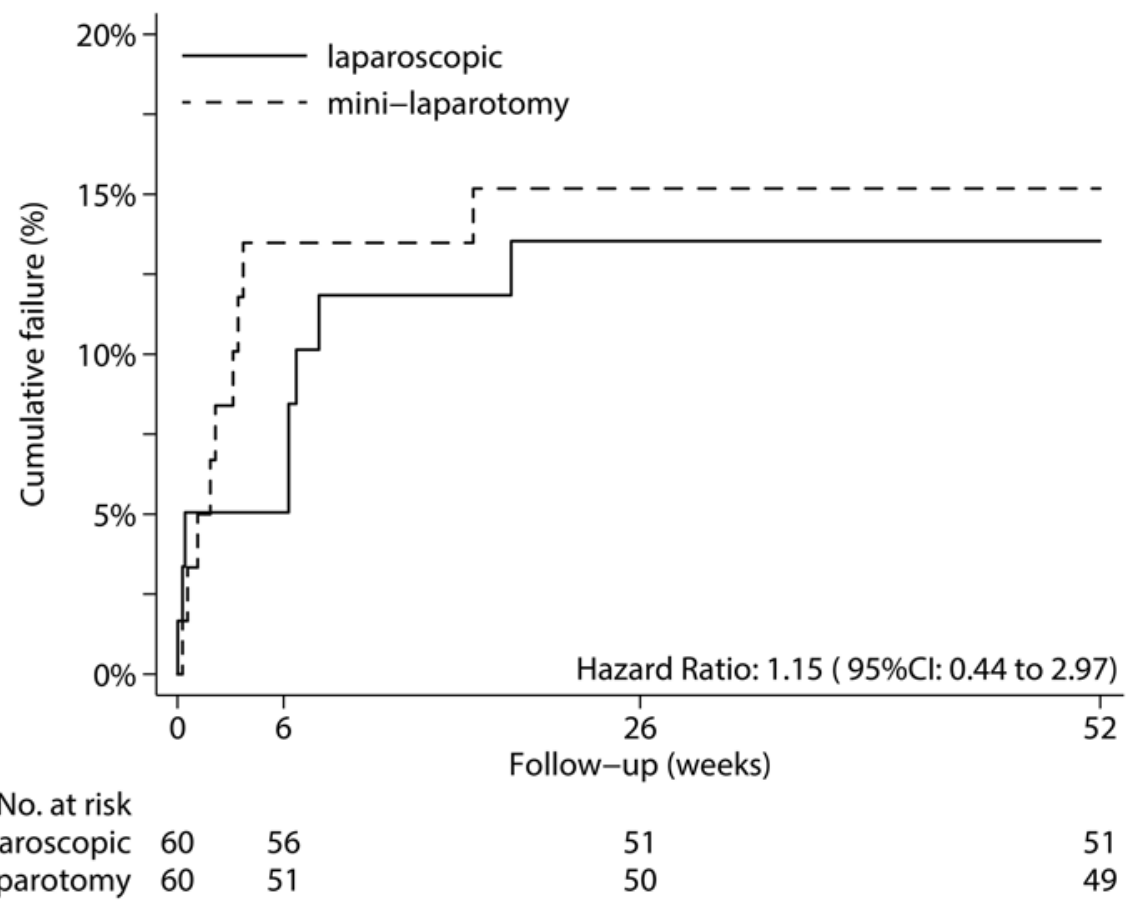

FIG. 3. Kaplan-Meier curve for cumulative overall shunt failure requiring surgical intervention. Overall shunt failure increased rapidly in the mini-laparotomy group within the first 6 postoperative weeks. However, at the 6-month and 12-month follow-up points the differences in the overall VP shunt failure rates between the mini-laparotomy and the laparoscopic groups were not statistically significant.

While a visceral (or general) surgeon will have more experience working in the abdominal cavity, it seems unlikely that the type of surgical training is responsible for the differences in distal shunt failure rates. Catheter displacement or misplacement (e.g., for placement of a peritoneal dialysis catheter) is a problem equally encountered by visceral surgeons. Still, the visceral surgeon's additional expertise in abdominal approaches might have influenced our results.

\section{Reasons for Different Distal Failure Rates}

Age, duration of surgery, BMI, and previous abdominal surgery had no statistically significant influence on the risk of distal shunt failure in our study. Roth et al. speculated that the decreased rate of distal shunt obstructions in their laparoscopic group (4\% vs $10.3 \%$ after mini-laparotomy) may have been due to less frequent dislocation out of the peritoneal cavity owing to a smaller peritoneal hole and lysing of adhesions during shunt placement. ${ }^{25}$ Dislocation of the distal catheter out of the peritoneal cavity or inadvertent ligation of the catheter at the entry point into the peritoneum were the causes for all 5 distal shunt failures in our mini-laparotomy group. The method of entry into the peritoneal cavity is, however, the precise difference between the two surgical approaches. Distal catheter placement via mini-laparotomy will always require a slightly larger peritoneal incision to control correct catheter placement. This inevitably results in the catheter entrance point requiring some form of purse-string (or similar) suture to secure its position. On the other hand, the laparoscopic approach using a peel-away needle only pierces the abdominal wall, necessitating no closure or additional catheter fixation.

\section{Infections}

Shunt infections are a frequent reason for shunt failure. Our overall infection rate of $3.3 \%$ (4 out of 120 patients) is in line with the range of infection rates of $1 \%-11 \%$ reported in the literature..$^{11,21,31}$

The influence of the laparoscopic shunt insertion technique on the rate of infection is debatable. Retrospective analyses have previously observed a trend toward either higher ${ }^{4,20}$ or lower ${ }^{25}$ infection rates with a laparoscopic approach to shunt implantations than with a mini-laparotomy approach. In these studies, the reported rates of infections in laparoscopic shunt insertions ranged from 1.3\% to $13.5 \%$, and the differences from infection rates after mini-laparotomy (range 0\%-12\%) remained statistically insignificant. Our prospective trial adds evidence that the use of laparoscopic guidance for shunt insertion does not significantly influence infection rates.

\section{Intraoperative Complications}

The overall rate of intraoperative complications was $1.7 \%$, with 2 patients in the laparoscopic group requiring a deviation from allocated surgery due to small bowel lesions. In both cases, the lesions were inflicted by experienced visceral surgeons during the initial preparation phase and prior to insertion of the Cystofix peel-away needle. Both patients had undergone previous abdominal surgery, as was the case for a third of all patients in our study (Table 3 ). Damage to intra-abdominal organs remains a risk of any approach to the abdomen. Indeed, a recently published systematic review and meta-analysis comparing open to laparoscopic adhesiolysis in patients with adhesive small bowel obstruction did not show an increase in intraoperative small 


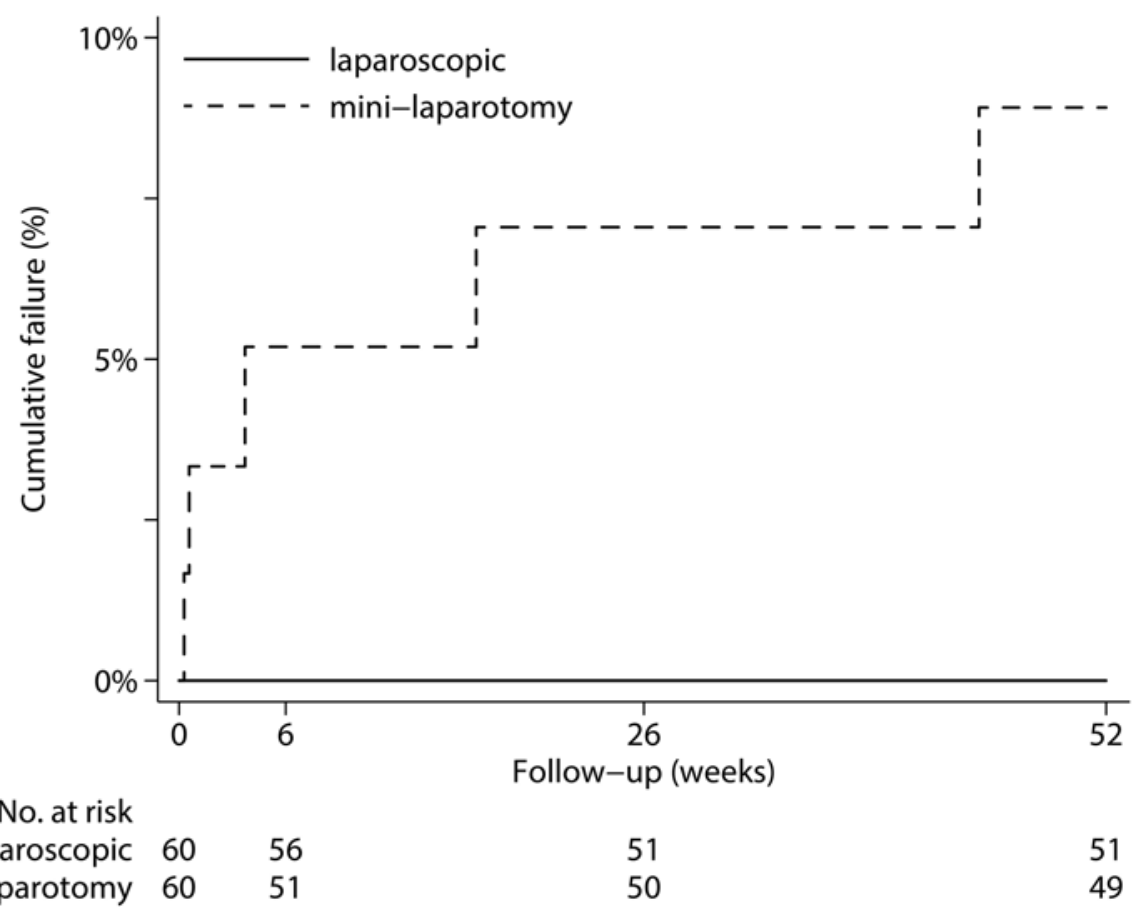

FIG. 4. Kaplan-Meier curve for cumulative distal shunt failure requiring surgical intervention. Distal shunt failure requiring surgical revision or shunt explantation occurred only in the mini-laparotomy group. No patient in the laparoscopic group required shunt revision for distal shunt failure.

bowel lesions in the laparoscopic group (OR 1.93, 95\% CI $0.76-4.89, \mathrm{p}=0.17) .{ }^{17}$ Laparoscopy-associated small bowel lesions remain rare; van der Voort and colleagues reported rates of $0.22 \%$ gastrointestinal perforation, half of which were due to small bowel lesions. ${ }^{32}$ Viscus perforation as a complication of laparoscopic VP shunt placement has previously been reported, ${ }^{29}$ but the risk seems to be moderate (the rate was $0 \%$ in the largest report, which involved 475 VP shunting procedures). ${ }^{20}$ Of note, the lesions were inflicted by senior visceral surgeons whose experience in approaches to the abdomen by far surpasses the neurosurgeons' experience. We nevertheless believe that it remains very important for the surgical team on site to make the final, patient-individualized decision as to whether the laparoscopic approach should be used.

\section{Duration of Surgery}

Although the difference was not statistically significant, we observed shorter operating times in the laparoscopic group. This may be partially explained by the presence of an additional surgeon during surgical procedures in the laparoscopic group, allowing simultaneous work on the abdominal and the cranial part of the surgery. Initially, the setup of the laparoscopic instruments required more time than expected, mainly because the neurosurgical theater staff was not familiar with the laparoscopic tools. This time lag vanished once the neurosurgical staff became familiar with the laparoscopic equipment. Availability of the laparoscopic surgeon was usually not an issue, as most patients were selected for randomization 1 day prior to the surgery, allowing for sufficient planning on behalf of the abdominal team. We had 1 single cross- over from the laparoscopic to the mini-laparotomy group due to unavailability of the visceral surgeon on call.

Whether laparoscopic VP shunt placement reduces or increases operative time remains a matter of debate. A definitive increase in time spent in the operating theater, mainly due to time needed for laparoscopic setup and waiting for the general surgeon, has previously been reported. ${ }^{27,31}$ On the other hand, in the largest series to date, Naftel et al. reported an overall reduction in operative times. ${ }^{20}$ The overall duration of surgery seems to depend on how familiar the neurosurgical theater staff is with laparoscopic instruments and on the availability of general surgeons. Thus we believe that a high caseload and good collaboration with colleagues from general surgery result in a reduction in the time required for surgery.

\section{Time to Regain Full Mobility, Analgesic Requirements, and Length of Stay}

In general, the advantages of laparoscopic surgery over mini-laparotomy include shorter hospital stay, reduced postoperative pain, and a shorter recovery time..$^{18,19,28}$ However, analyses of our patients' postoperative hospital stays, time to recovery of full mobility, and analgesic requirements did not reveal significant differences between the 2 groups (e.g., mean hospital stays were 8.9 days for the mini-laparotomy group and 9.6 days for the laparoscopic group). This incongruence is likely due to patient selection; the majority of our patients required a VP shunt for bleeding- or tumor-related hydrocephalus. In this setting, hospital stay is inevitably governed and prolonged by the underlying illness and thus largely independent of the technique used for distal catheter placement. The postoperative 
TABLE 3. Evaluation of factors potentially responsible for increased risk of distal shunt failure requiring surgical intervention*

\begin{tabular}{lccc}
\hline \multicolumn{1}{c}{ Study Parameter } & $\begin{array}{c}\text { Patients w/ Distal Shunt } \\
\text { Failure }(\mathrm{n}=5)\end{array}$ & $\begin{array}{c}\text { Patients w/o Distal Shunt } \\
\text { Failure }(\mathrm{n}=115)\end{array}$ & $\mathrm{p} \mathrm{Value \dagger}$ \\
\hline Mean age $(\mathrm{yrs})$ & $64.2 \pm 10.3$ & $58.0 \pm 16.2$ & 0.40 \\
\hline Mean duration of surgery (minutes) & $69.0 \pm 18.5$ & $68.0 \pm 33.9$ & 0.95 \\
\hline Mean BMl $\left(\mathrm{kg} / \mathrm{m}^{2}\right)$ & $27.0 \pm 4.8$ & $25.5 \pm 4.9$ & 0.49 \\
\hline Previous abdominal surgery & & $76(66 \%)$ & 1 \\
\hline No & $4(80 \%)$ & $38(33 \%)$ & \\
\hline Yes & $1(20 \%)$ & $1(1 \%)$ & \\
\hline Unknown & $0(0 \%)$ & & \\
\hline * Values represent numbers of patients $(\%)$ unless otherwise indicated. All patients with distal shunt failure were in the mini-laparotomy group. \\
$\dagger$ Student's t-test for continuous outcomes; Fisher's exact test for categorical variables.
\end{tabular}

analgesic requirement 5 days after surgery was not significantly affected by mode of distal catheter placement, as most patients required regular analgesia for pain that was not related to the site of peritoneal catheter placement.

\section{Conclusions}

Reasons for VP shunt failure are numerous, and it seems unlikely that a single change of protocol will ever resolve the issue entirely. Our study reveals a significant reduction in distal catheter malfunction or failure for laparoscopic shunt insertion compared with mini-laparotomy $(\mathrm{p}=0.03)$. However, the effect of this distal failure reduction was diluted in the rate of overall shunt failures $(p=0.4)$. Reasons for proximal malfunction, such as incorrect positioning of a ventricular catheter, reduce this positive effect to a trend toward fewer shunt failures with laparoscopic shunt insertion than with mini-laparotomy. Another potential advantage of the laparoscopic approach lies in a trend toward fewer

TABLE 4. Summary of all evaluated secondary endpoints*

\begin{tabular}{|c|c|c|c|}
\hline \multirow[b]{2}{*}{ Study Parameter } & \multicolumn{2}{|c|}{ Group } & \multirow[b]{2}{*}{$\mathrm{p}$ Value } \\
\hline & Laparoscopic $(n=60)$ & Mini-Laparotomy $(n=60)$ & \\
\hline Mean duration of surgery (minutes) & $63.9 \pm 35.8$ & $72.2 \pm 30.5$ & 0.179 \\
\hline Mean time until full mobility (days) & $5.5 \pm 2.2$ & $5.4 \pm 2.3$ & 0.903 \\
\hline Mean time until solid food intake (days) & $4.0 \pm 2.2$ & $4.4 \pm 2.1$ & 0.308 \\
\hline Mean length of stay (days) & $9.6 \pm 11.9$ & $8.9 \pm 9.4$ & 0.715 \\
\hline Shunt dysfunction & & & 0.053 \\
\hline None & $54(90 \%)$ & $49(82 \%)$ & \\
\hline Proximal & $5(8 \%)$ & $2(3.3 \%)$ & \\
\hline Infection & $1(1.7 \%)$ & $3(5 \%)$ & \\
\hline Distal & $0(0 \%)$ & $5(8 \%)$ & \\
\hline Not assessed & $0(0 \%)$ & $1(2 \%)$ & \\
\hline Correct intraventricular catheter position & & & 0.244 \\
\hline Yes & $57(95 \%)$ & $60(100 \%)$ & \\
\hline No & $2(3 \%)$ & $0(0 \%)$ & \\
\hline Not assessed & $1(2 \%)$ & $0(0 \%)$ & \\
\hline Abdominal catheter kinking & & & 0.496 \\
\hline No & $58(97 \%)$ & $60(100 \%)$ & \\
\hline Not assessed & $2(3 \%)$ & $0(0 \%)$ & \\
\hline Correct intraperitoneal catheter position & & & 0.496 \\
\hline Yes & $58(97 \%)$ & $60(100 \%)$ & \\
\hline Not assessed & $2(3 \%)$ & $0(0 \%)$ & \\
\hline Use of NSAIDs on Day 5 & & & 0.364 \\
\hline Yes & $27(45 \%)$ & $26(43 \%)$ & \\
\hline No & $26(43 \%)$ & $31(52 \%)$ & \\
\hline Not assessed & $7(12 \%)$ & $3(5 \%)$ & \\
\hline
\end{tabular}


infections and shorter operation times in the laparoscopic cohort. In larger hospitals where availability of general surgeons is not an issue, laparoscopic VP shunt placement is an elegant, feasible alternative to the standard mini-laparotomy approach and can decrease the rate of distal shunt failures. However, careful patient selection is warranted to minimize the risk of gastrointestinal perforations.

\section{Acknowledgment}

We thank Daniela Miescher for graphic design.

\section{References}

1. Abu-Dalu K, Pode D, Hadani M, Sahar A: Colonic complications of ventriculoperitoneal shunts. Neurosurgery 13:167169,1983

2. Ames RH: Ventriculo-peritoneal shunts in the management of hydrocephalus. J Neurosurg 27:525-529, 1967

3. Bani A, Hassler WE: Laparoscopy-guided insertion of peritoneal catheters in ventriculoperitoneal shunt procedures: analysis of 39 children. Pediatr Neurosurg 42:156-158, 2006

4. Bani A, Telker D, Hassler W, Grundlach M: Minimally invasive implantation of the peritoneal catheter in ventriculoperitoneal shunt placement for hydrocephalus: analysis of data in 151 consecutive adult patients. J Neurosurg 105:869-872, 2006

5. Basauri L, Selman JM, Lizana C: Peritoneal catheter insertion using laparoscopic guidance. Pediatr Neurosurg 19:109-110, 1993

6. Beningfield S, Potgieter H, Nicol A, van As S, Bowie G, Hering E, et al: Report on a new type of trauma full-body digital X-ray machine. Emerg Radiol 10:23-29, 2003

7. Cuatico W, Vannix D: Laparoscopically guided peritoneal insertion in ventriculoperitoneal shunts. J Laparoendosc Surg 5:309-311, 1995

8. Fanelli RD, Mellinger DN, Crowell RM, Gersin KS: Laparoscopic ventriculoperitoneal shunt placement: a single-trocar technique. Surg Endosc 14:641-643, 2000

9. Grosfeld JL, Cooney DR, Smith J, Campbell RL: Intra-abdominal complications following ventriculoperitoneal shunt procedures. Pediatrics 54:791-796, 1974

10. Hasson HM: Open laparoscopy. Biomed Bull 5:1-6, 1984

11. Kanev PM, Sheehan JM: Reflections on shunt infection. Pediatr Neurosurg 39:285-290, 2003

12. Kausch W: Die behandlung des hydrocephalus der kleinen Kinder. Arch Klin Chir 87:709-796, 1908

13. Khaitan L, Brennan EJ Jr: A laparoscopic approach to ventriculoperitoneal shunt placement in adults. Surg Endosc 13:1007-1009, 1999

14. Khosrovi H, Kaufman HH, Hrabovsky E, Bloomfield SM, Prabhu V, el-Kadi HA: Laparoscopic-assisted distal ventriculoperitoneal shunt placement. Surg Neurol 49:127-135, 1998

15. Kirshtein B, Benifla M, Roy-Shapira A, Merkin V, Melamed I, Cohen Z, et al: Laparoscopically guided distal ventriculoperitoneal shunt placement. Surg Laparosc Endosc Percutan Tech 14:276-278, 2004

16. Kurschel S, Eder HG, Schleef J: CSF shunts in children: endoscopically-assisted placement of the distal catheter. Childs Nerv Syst 21:52-55, 2005

17. Li MZ, Lian L, Xiao LB, Wu WH, He YL, Song XM: Laparoscopic versus open adhesiolysis in patients with adhesive small bowel obstruction: a systematic review and meta-analysis. Am J Surg 204:779-786, 2012

18. Marks JH, Kawun UB, Hamdan W, Marks G: Redefining contraindications to laparoscopic colorectal resection for high-risk patients. Surg Endosc 22:1899-1904, 2008

19. Maurus CF, Schäfer M, Müller MK, Clavien PA, Weber M: Laparoscopic versus open splenectomy for nontraumatic diseases. World J Surg 32:2444-2449, 2008

20. Naftel RP, Argo JL, Shannon CN, Taylor TH, Tubbs RS, Cle- ments RH, et al: Laparoscopic versus open insertion of the peritoneal catheter in ventriculoperitoneal shunt placement: review of 810 consecutive cases. Clinical article. J Neurosurg 115:151-158, 2011

21. Parker SL, Attenello FJ, Sciubba DM, Garces-Ambrossi GL, Ahn E, Weingart J, et al: Comparison of shunt infection incidence in high-risk subgroups receiving antibiotic-impregnated versus standard shunts. Childs Nerv Syst 25:77-85, 2009

22. Raimondi AJ: Hydrocephalus, in: Pediatric Neurosurgery: Theoretical Principles and Surgical Techniques. New York: Springer Verlag, 1987, pp 453-491

23. Raimondi AJ, Matsumoto S: A simplified technique for performing the ventriculo-peritoneal shunt. Technical note. J Neurosurg 26:357-360, 1967

24. Reardon PR, Scarborough TK, Matthews BD, Marti JL, Preciado A: Laparoscopically assisted ventriculoperitoneal shunt placement using 2-mm instrumentation. Surg Endosc 14:585-586, 2000

25. Roth J, Sagie B, Szold A, Elran H: Laparoscopic versus nonlaparoscopic-assisted ventriculoperitoneal shunt placement in adults. A retrospective analysis. Surg Neurol 68:177-184, 2007

26. Schievink WI, Wharen RE Jr, Reimer R, Pettit PD, Seiler JC, Shine TS: Laparoscopic placement of ventriculoperitoneal shunts: preliminary report. Mayo Clin Proc 68:1064-1066, 1993

27. Schubert F, Fijen BP, Krauss JK: Laparoscopically assisted peritoneal shunt insertion in hydrocephalus: a prospective controlled study. Surg Endosc 19:1588-1591, 2005

28. Schwenk W, Haase O, Neudecker JJ, Müller JM: Short term benefits for laparoscopic colorectal resection. Cochrane Database Syst Rev (3):CD003145, 2005

29. Stoddard T, Kavic SM: Laparoscopic ventriculoperitoneal shunts: benefits to resident training and patient safety. JSLS 15:38-40, 2011

30. Therneau TM, Grambsch PM: Modeling Survival Data: Extending the Cox Model. New York: Springer, 2000

31. Turner RD, Rosenblatt SM, Chand B, Luciano MG: Laparoscopic peritoneal catheter placement: results of a new method in 111 patients. Neurosurgery 61 (3 Suppl):167-174, 2007

32. van der Voort M, Heijnsdijk EA, Gouma DJ: Bowel injury as a complication of laparoscopy. Br J Surg 91:1253-1258, 2004

33. Wilson CB, Bertan V: Perforation of the bowel complicating peritoneal shunt for hydrocephalus. Report of two cases. Am Surg 32:601-603, 1966

34. Yu S, Bensard DD, Partrick DA, Petty JK, Karrer FM, Hendrickson RJ: Laparoscopic guidance or revision of ventriculoperitoneal shunts in children. JSLS 10:122-125, 2006

\section{Author Contributions}

Conception and design: Schucht, Trochsler, Kuhlen, Mariani. Acquisition of data: Schucht, Trochsler, Krähenbühl, Reinert, Beck, Raabe, Candinas, Kuhlen, Mariani. Analysis and interpretation of data: Schucht, Banz, Iff. Drafting the article: Schucht, Banz. Critically revising the article: Schucht, Banz, Trochsler, Krähenbühl, Reinert, Beck, Raabe, Candinas. Reviewed submitted version of manuscript: all authors. Approved the final version of the manuscript on behalf of all authors: Schucht. Statistical analysis: Iff.

\section{Supplemental Information \\ Current Affiliation}

Dr. Kuhlen: Department of Neurosurgery, Ospedale Cantonale di Lugano, Switzerland.

\section{Correspondence}

Philippe Schucht, Department of Neurosurgery, Bern University Hospital, Freiburgstrasse 10, 3010 Bern, Switzerland. email: philippe.schucht@insel.ch. 